\title{
Heating behavior of magnetic iron oxide nanoparticles at clinically relevant concentration
}

\author{
Philipp Lemal ${ }^{\mathrm{a}}$, Sandor Balog ${ }^{\mathrm{a}}$, Christoph Geers ${ }^{\mathrm{a}}$, Patricia Taladriz-Blanco ${ }^{\mathrm{a}}$, Andrea Palumbo ${ }^{\mathrm{a}}$, \\ Ann M. Hirt ${ }^{\mathrm{b}}$, Barbara Rothen-Rutishauser ${ }^{\mathrm{a}}$, Alke Petri-Fink ${ }^{\mathrm{a}, \mathrm{c}, *}$ \\ adolphe Merkle Institute, University of Fribourg, Chemin des Verdiers 4, CH-1700 Fribourg, Switzerland \\ ${ }^{\mathrm{b}}$ Institute for Geophysics, ETH Zurich, Sonneggstrasse 5, CH-8092 Zurich, Switzerland \\ ${ }^{\mathrm{C}}$ Chemistry Department, University of Fribourg, Chemin du Museé 9, CH-1700 Fribourg Switzerland
}

\section{A R T I C L E I N F O}

\section{Keywords:}

High concentration

Magnetic nanoparticles

Hyperthermia

- Nanoparticle characterization

\begin{abstract}
A B S T R A C T
Magnetic hyperthermia for cancer treatment has gained significant attention in recent years, due to its biocompatibility of applied nanoparticles and the possibility for spatially localized heating in deep tissues. Clinical treatments use nanoparticle concentrations of $112 \mathrm{mg} \mathrm{Fe} / \mathrm{mL}$, while the concentrations experimental studies have addressed are considerably smaller, usually between 0.1 and $30 \mathrm{mg} / \mathrm{mL}$. Therefore, it is not clear whether such experiments correspond to the magnetic properties found in clinical applications. In this regard, we studied the thermal behavior of superparamagnetic iron oxide nanoparticles (SPION) with the most common particle shapes used in the field, including spherical (core diameters 11 and $19 \mathrm{~nm})$, cubic $(15 \mathrm{~nm})$ and ellipsoidal (23 nm with an aspect ratio of 1.45), at concentrations ranging from 5 to $80 \mathrm{mg} \mathrm{Fe} / \mathrm{mL}$. Their shape, size, crystallinity, magnetic, and thermal behavior were characterized via transmission electron microscopy, dynamic light scattering, Taylor dispersion analysis, X-ray diffraction, alternating gradient magnetometry, and lock-in thermography. Spherical and cubic nanoparticles displayed linear heating slopes, independent from size, shape and concentration, resulting in unchanged specific absorption rates (SAR). Ellipsoids showed the same behavior until $50 \mathrm{mg} / \mathrm{mL}$, above which a decreasing heating slope trend was found, without evidence as to what causes this behavior. However, the presented results highlight the importance of colloidally stable SPIONs in magnetic hyperthermia to obtain maximum heating power by minimum particle dosage.
\end{abstract}

\section{Introduction}

Magnetic hyperthermia is a therapeutic approach using magnetic nanoparticles (MNPs) to heat cancerous tissue above normal physiological conditions by converting externally-supplied magnetic energy into thermal energy [1]. What makes this method interesting is the superparamagnetic behavior of the particles, biocompatibility, possibility of targeted delivery of the particles, and the spatially localized heating in deep tissues due to an applied alternating magnetic field (AMF) [2]. The heating efficiency of MNPs relies on external characteristics (e.g. frequency and amplitude of the applied field) as well as intrinsic particle characteristics (e.g. core size, surface chemistry, crystallinity, colloidal) [2-7]. The term magnetic hyperthermia was first introduced by Gilchrist et al. [8] in 1957, and has received increasing interest ever since the 1980s [9]. In fact, hyperthermia-based and nanoparticlemediated adjuvant therapy for glioblastoma multiforme received the first and only European clinical approval in 2011 [10]. NanoTherm ${ }^{\circ}$ therapy uses an aqueous dispersion of superparamagnetic nanoparticles with an iron concentration of $112 \mathrm{mg} / \mathrm{mL}$ [10]. As a consequence of the high concentration, the smaller inter-particle distance may lead to increasing magnetic dipole-dipole interactions and to the formation of agglomerates/aggregates. Both phenomena reduce the heating efficiency of the particles due to hindered relaxation processes $[2,11]$. In contrast with the high concentration used in clinical practice, typical iron concentrations tested in research laboratories range from 0.1 to $30 \mathrm{mg} / \mathrm{mL}[12-20]$. As a consequence, such test results-although intended for providing clinical insight-might be of little relevance. While there is a powerful model by Rosensweig [4] that explains the heating efficiency of individual, stable and non-interacting magnetic nanoparticles, the community has still not agreed on a model dedicated to describe highly concentrated particle suspension [2]. To date, the models of Dormann et al. [21] and Mørup and Tronc [22] explain the effect of increasing dipolar interactions. However, they are contradictory: Dormann's model predicts that with increasing particle

\footnotetext{
* Corresponding author at: Adolphe Merkle Institute, University of Fribourg, Chemin des Verdiers 4, CH-1700 Fribourg, Switzerland.

E-mail address: alke.fink@unifr.ch (A. Petri-Fink).
} 
concentration the Néel relaxation time increases, whereas Mørup and Tronc's model estimates the exact opposite. How this influences the heating efficiency of highly concentrated particles seems to be dependent on the dominant relaxation mechanism [2]. Briefly, when increasing the concentration, Néel-dominated particle systems exhibit a decrease in the specific absorption rate [23-26], a value representing a commonly used intrinsic heat generation value [27]. Brownian-dominated MNPs appear to have increasing SAR values at higher concentrations $[20,24,28,29]$. However, these studies were conducted at the low concentrations described above. As one of the few studies investigating higher concentrations, Ota et al. [30] could determine the concentration effect for water-dispersed MNPs between 37 and $370 \mathrm{mg}$ $\mathrm{Fe} / \mathrm{mL}$. By dc and ac hysteresis loop measurements, they could show that magnetization, and thus the heating power, decreases with increasing concentration due to stronger dipole-dipole interactions. However, they could not provide detailed information about the state of colloidal stability for their samples at higher concentration. Overall one can say that the origin for changes in magnetic properties upon increased particle concentration is not yet clarified and more experimental data is needed.

The aim of this study was to investigate the thermal behavior of highly concentrated but colloidally stable particles, to monitor concentration effects on SAR values uninfluenced by aggregates. We synthesized SPIONs batches varying in size and shape by thermal decomposition, transferred them to an aqueous environment by ligand exchange with citric acid (CA), and stabilized in a concentration range between 5 and $80 \mathrm{mg} \mathrm{Fe} / \mathrm{mL}$. Colloidal stability was evaluated by alternating gradient magnetometer and Taylor dispersion analysis (TDA), while thermal measurements were conducted with a previously described lock-in thermography set-up [31].

\section{Experimental section}

\subsection{Chemicals}

Iron (III) chloride hexahydrate of ACS reagent grade was purchased from Sigma-Aldrich, Merck KGaA or Acros Organics. Sodium oleate $(>97 \%)$ and tri-n-octylamine ( $>97 \%$ ) were supplied by TCI chemicals. Absolute ethanol (100\%) and n-hexane (98\%) of ACS reagent grade were purchased from VWR Chemicals. Sigma-Aldrich supplied oleic acid (90\%, technical grade), 1,2-dichlorobenzene (DCB, 99\%) and n,n-Dimethylformamide (DMF, $\geq 99.8 \%$ ). Diethyl ether (ACS reagent grade) and ammonium hydroxide solution (25\%) were obtained from Honeywell Burdick \& Jackson. Acetone (technical grade) was supplied by Reactolab SA. All chemicals were used as received without any further purification. Each aqueous solution was prepared with deionized water received from a Milli-Q system (resistivity $=18.2 \mathrm{M} \Omega \mathrm{cm}$, Millipore AG).

\subsection{Synthesis of SPIONs, ligand exchange and colloidal stabilization}

The SPIONs used in this work were synthesized by thermal decomposition according to a modified procedure reported by Park et al. [32] and Sun and Zeng [33]. The desired NP sizes were achieved synthetically by changing the heating ramps $\left(30-130^{\circ} \mathrm{C}\right.$ at $10^{\circ} \mathrm{C} / \mathrm{min}$; $130-320^{\circ} \mathrm{C}$ at $3{ }^{\circ} \mathrm{C} / \mathrm{min} ; 1 \mathrm{~h}$ at $320^{\circ} \mathrm{C}$ ), amount of iron (III) chloride hexahydrate $(32 \mathrm{mmol}$ ), and minor variation in iron oleate complex proportion to oleic acid. The specific shapes could be varied by introducing different amounts of sodium oleate into the reaction [34]. To transfer the SPIONs in aqueous solutions a ligand exchange with CA was performed [35]. Colloidal stability at high concentration was ensured by adding an excess of unbound CA into the dispersions and subsequent adjustment to $\mathrm{pH} 7-8$ [36].

\subsection{Transmission electron microscopy}

The size, dispersity, and morphology of the synthesized SPIONs were analyzed by transmission electron microscopy (TEM). Diluted samples were drop casted onto 300 mesh carbon membrane-coated copper grids following a procedure described by Michen and Geers et al. [37]. TEM experiments were carried out on a FEI Tecnai Spirit operating at a voltage of $120 \mathrm{kV}$ and equipped with a side-mounted Veleta CCD camera (Olympus). The core diameters of the nanoparticles were determined using an automatized size distribution analysis macro in ImageJ (v1.50i).

\subsection{Dynamic light scattering}

Dynamic light scattering (DLS) measurements were carried out at $25^{\circ} \mathrm{C}$ using a commercial goniometer instrument (3D LS Spectrometer, LS Instruments AG, Switzerland). The laser beam was linearly polarized (Cobolt 05-01 diode pumped solid-state laser, $\lambda=660 \mathrm{~nm}, P$ max. $=500 \mathrm{~mW}$ ), and a single-mode optical fibre equipped with integrated collimation optics collected the scattered light, which also passed through a laser-line filter. Measurements were made at scatterings angles between $60^{\circ}$ and $120^{\circ}$, and the autocorrelation functions were analysed as described elsewhere [38].

\subsection{Iron quantification by ICP-OES}

Inductively coupled plasma optical emission spectroscopy (ICP-OES, PerkinElmer Avio 200) was used to quantify the iron concentration in all suspensions. Each NP dispersion $(10 \mu \mathrm{L})$ was dissolved in aqua regia $\left(\mathrm{HNO}_{3}: \mathrm{HCl}\right.$ with a volume ratio of $1: 3,1 \mathrm{~mL}$ per tube), dissolved overnight and diluted to $10 \mathrm{~mL}$ with Milli-Q water the next day. Furthermore, a standardized aqueous iron solution (TraceCERT ${ }^{\oplus}$, Fluka Analytical) was used to prepare a calibration curve and all samples were measured by ICP-OES at a wavelength of $238.2 \mathrm{~nm}$ for iron.

\subsection{Alternating gradient magnetometer}

Magnetization curves of the citric acid coated SPIONs were measured by an alternating gradient magnetometer (MicroMag Model 2900, Princeton Measurements Corporation). NP suspensions of low and high iron concentrations were injected into hydrophobic cotton pellets and dried overnight. The samples were fixed to the sample holder with grease and hysteresis loop measurements were performed at a constant field increment, using a measurement averaging time of $100 \mathrm{~ms}$ at room temperature. Normalizing the obtained magnetization profiles by the dried sample mass of iron results in the final magnetization curves.

\subsection{Thermal measurements}

The thermal signal of each particle suspension was analyzed with a lock-in thermal imaging setup described by Monnier et al. [31]. Briefly, an alternating magnetic field is generated by a commercial coil system (Magnetherm ${ }^{\text {TM }}$ V1.5, nanoTherics Ltd) consisting of a water cooled coil, a laboratory power supply (EA-PS 3032-20B, EA Elektro Automatic) and a function generator (SFG-2004, GW Instek). All measurements were carried out at $523 \mathrm{kHz}$ and $14.7 \mathrm{kA} / \mathrm{m}$. Detailed information about the magnetic field where described elsewhere [39]. The thermal signals were captured using an infrared camera (Onca-MWIR-InSb-320, XenICs) which recorded infrared radiations with a full frame rate of $250 \mathrm{~Hz}$. Data were transferred to a personal computer and processed in real time with a modulation frequency of $1.0 \mathrm{~Hz}$ for all measurements. The SAR value was calculated based on the initial heating slope $(\beta$, i.e. $\Delta$ temperature/ $\Delta$ time) taking the heat capacity of the solution $\left(c_{\mathrm{p}}\right)$ as well as the mass of magnetic material inside $\left(\mathrm{m}_{\mathrm{FE}}\right)$ into account. 
$S A R=\boldsymbol{\beta} \times \frac{c_{p}}{m_{F e}}$

\subsection{Taylor dispersion analysis}

Taylor dispersion analyses were carried out as described elsewhere [40]. Taylorgrams were collected using a capillary electrophoresis injection system (Prince $560 \mathrm{CE}$ Autosampler, Prince Technologies B.V.) applying a fused silica capillary $(74.5 \mu \mathrm{m}$ inner diameter, Polymicro Technologies, Phoenix, USA) at constant temperature of $25^{\circ} \mathrm{C}$. Data was collected by an ActiPix D100 UV-Vis area imaging detector (Paraytec, York, UK, $20 \mathrm{~Hz}$ sampling rate). Time-resolved extinction was measured using a band-pass filter (center wavelength of $520 \mathrm{~nm}$ ) coupled with a neutral density filter ( $10 \%$ transmission, Edmund Optics, York, UK). The running buffer for all measurements was MilliQ-water used under continuous flow conditions of 90 mbar to drive the samples trough the capillary. The full length of the capillary was $145.5 \mathrm{~cm}$, equipped with two detection windows with a width of $1 \mathrm{~cm}$ each (length to first window: $72.5 \mathrm{~cm}$; length to second window: $104.5 \mathrm{~cm}$ ). The instrument automatically corrected the intensity values for dark current, controls the intensity of illumination and perform a background measurement of the respective running buffer before each run. Consequently, the extinction of the particles is expected to be collected in the linear range of the detector throughout the measurements, and the resulting taylorgram is determined by taking into account solvent backgrounds, dark current, and variations in the illumination.

\subsection{Zeta potential measurements}

The effective surface charge was determined at $25^{\circ} \mathrm{C}$ using a Brookhaven ZetaPALS and estimated via the Henry equation using the Smoluchowski approximation [41]. Measurements were carried out using $50 \mu \mathrm{g} \mathrm{mL}^{-1}$ SPIONs in Milli-Q/PBS mixture and forty-five cycles of electrophoretic mobility measurements were performed ten times.
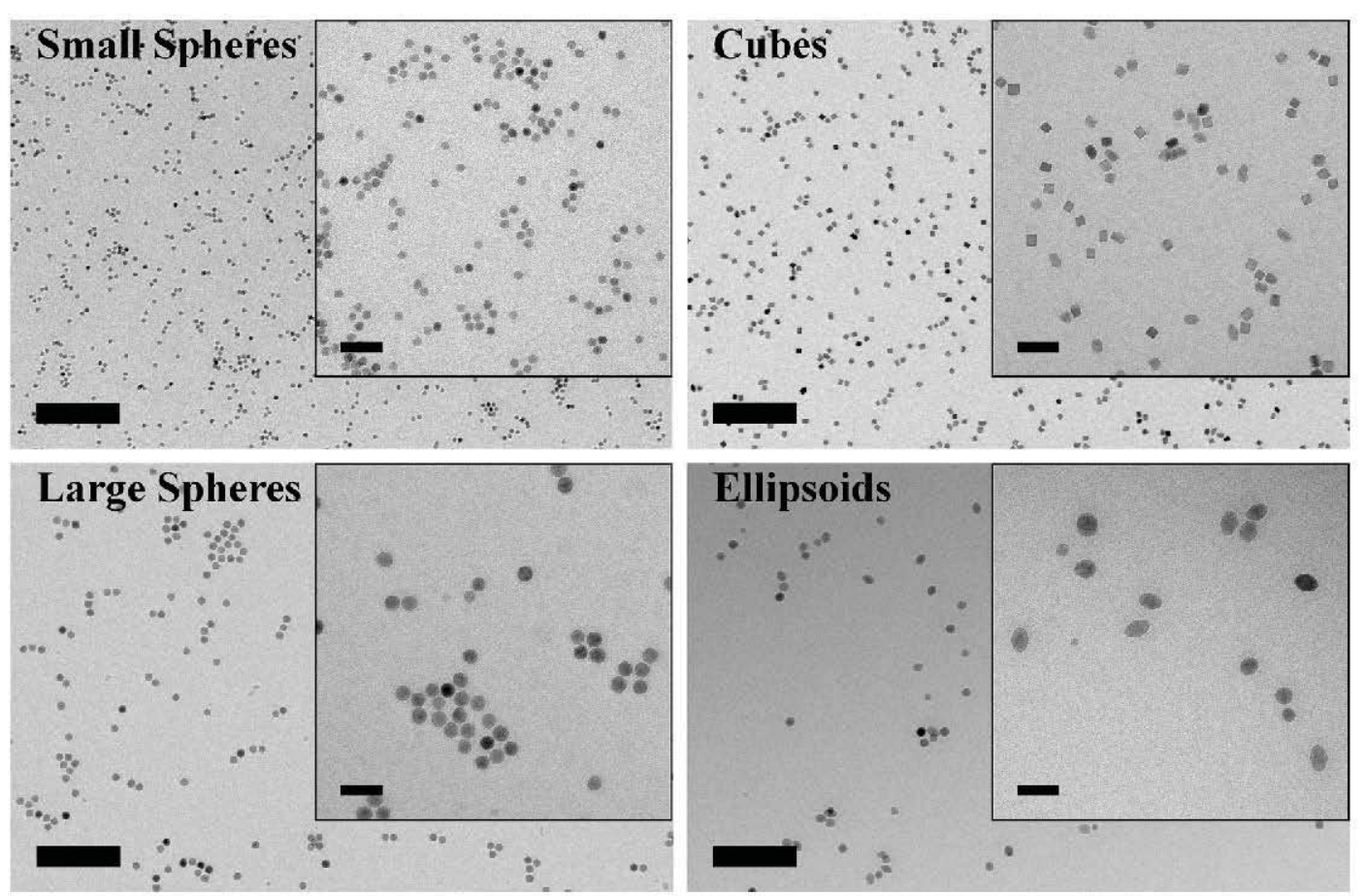

Fig. 1. TEM micrographs of CA coated SPIONs. All particles were synthesized by thermal decomposition and were transferred to water by ligand exchange. Scale bar $=200 \mathrm{~nm}$, insets $=50 \mathrm{~nm}$.

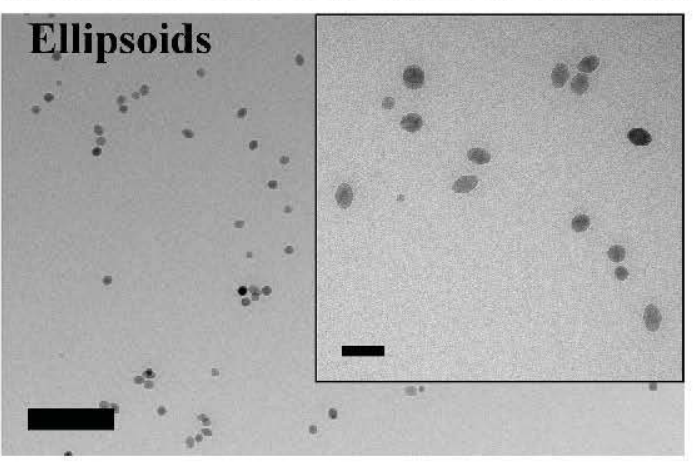

\subsection{X-ray diffraction}

X-ray diffraction (XRD) measurements were performed on an Ultima IV X-Ray diffractometer from Rigaku. Samples were dried on a glass slide and measurement range was set from $20^{\circ}$ to $80^{\circ}$. The resulting diffractograms were normalized to have the same maximum peak height.

\section{Results and discussion}

All SPIONs of this study were synthesized by thermal decomposition of an iron oleate complex [32,33], followed by a ligand exchange with CA to transfer them to water [35]. Colloidal stability even at higher concentrations was achieved by deprotonating CA hydroxyl groups in the presence of ammonia solution and subsequently adding unbound $\mathrm{CA}$, while adjusting the $\mathrm{pH}$ of the dispersion to 7-8 [36]. We studied the thermal behavior of the most common particle shapes used in the field, including two spherical (core diameters 11 and $19 \mathrm{~nm}$ ), one cubic $(15 \mathrm{~nm})$ and one ellipsoidal ( $23 \mathrm{~nm}$ with an aspect ratio of 1.45$)$ SPIONbatch which can be seen on the TEM micrographs in Fig. 1. Particle size was tuned by slight variation to precursor concentrations in the reaction, cubic and ellipsoidal SPION were obtained by addition of different amounts of sodium oleate to the thermal decomposition process $[34,42,43]$. Hydrodynamic diameters $\left(d_{H}\right)$ measured at low concentrations were found to show a slight size increase due to the presence of coating molecules, formation of a hydration shell and the electric double layer around the surface [44]. Considering these effects, DLS measurements (ranging from 19.6 to $29.4 \mathrm{~nm}$, see Table 1) are in good agreement with the TEM values, showing that SPIONs were colloidally stable at low concentration in water. Zeta potential measurements were found to be between -22 and $-28 \mathrm{mV}$ (see Table 1), explaining the colloidal stability in aqueous environment at low concentrations for all particle types. To the up-concentrated samples, we added free $\mathrm{CA}$ to further increase repulsive forces between the SPIONs, and thus, ensure high colloidal stability.

To evaluate whether the particles are colloidally stable at high concentrations, size measurements at the lowest and the highest 
Table 1

Summary of size and Zeta potential measurements of SPIONs (mean \pm standard deviation). Core diameters were determined by TEM, hydrodynamic diameters $\left(\mathrm{d}_{\mathrm{H}}\right)$ of diluted samples were measured by DLS, while TDA was used to determine $\mathrm{d}_{\mathrm{H}}$ at the lowest and highest concentration for each particle type. The Zeta potential was measured at a pH of 7.4 in MilliQ/PBS mixture. "TEM measurements of ellipsoids are shown with the mean major and minor diameter resulting in a mean aspect ratio of 1.45 .

\begin{tabular}{|c|c|c|c|c|c|}
\hline Particle type & $\mathrm{d}_{\mathrm{TEM}}(\mathrm{nm})$ & $\mathrm{d}_{H, D L S}(\mathrm{~nm})$ & $\mathrm{d}_{\mathrm{H}, \mathrm{TDS}}(\mathrm{nm})$ lowest concentration & $\mathrm{d}_{H, \mathrm{TDA}}(\mathrm{nm})$ highest concentration & Zeta potential (mV) \\
\hline Small Spheres & $11.1 \pm 2.4$ & $19.6 \pm 0.4$ & $11.2 \pm 0.7$ & $15.5 \pm 2.9$ & $-27.7 \pm 1.3$ \\
\hline Cubes & $15.3 \pm 1.3$ & $20.0 \pm 0.2$ & $11.7 \pm 3.5$ & $13.6 \pm 0.5$ & $-26.0 \pm 1.4$ \\
\hline Large Spheres & $19.0+3.3$ & $23.8 \pm 0.2$ & $19.9 \pm 0.6$ & $17.1 \pm 1.9$ & $-25.0 \pm 1.0$ \\
\hline \multirow[t]{2}{*}{ Ellipsoids } & $22.3 \pm 7.2$ & $29.4 \pm 4.6$ & $22.6 \pm 0.7$ & $22.1 \pm 0.6$ & $-21.6 \pm 1.9$ \\
\hline & $15.4 \pm 4.7$ & & & & \\
\hline
\end{tabular}
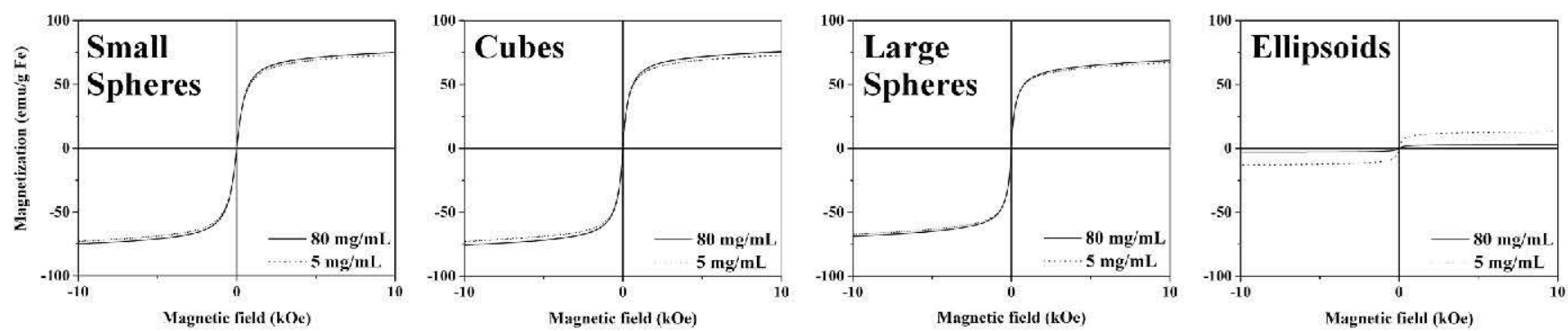

Fig. 2. Magnetization $M H$ curve comparison between 5 and $80 \mathrm{mg} / \mathrm{mL}$. Alternating gradient magnetometry highlighted the superparamagnetic nature of the SPIONs. Furthermore small spheres, cubes and large SPIONs showing similar curves for both concentrations. Ellipsoidal SPIONs exhibit overall a lower saturation magnetization at $5 \mathrm{mg} / \mathrm{mL}$, which further decreases drastically at $80 \mathrm{mg} / \mathrm{mL}$.

concentration were needed. However, DLS is not suitable to measure SPIONs at concentrations up to $80 \mathrm{mg} / \mathrm{mL}$. TEM is also limited to diluted samples, simply because high concentration result in undistinguishable lumps of particles. We therefore decided to apply another characterization technique, Taylor dispersion analysis, which was suitable to characterize the hydrodynamic diameters at high concentrations, without the need to dilute the samples beforehand, using very small sample volumes (nanoliters to microliters) [40]. Therefore, we were able to determine the hydrodynamic diameter of cubes, large spheres, and ellipsoids at initial concentrations of 5 and $80 \mathrm{mg} / \mathrm{mL} .100$ and $585 \mathrm{mg} / \mathrm{mL}$ samples were measured for small spheres. All particles were found to have a stable $\mathrm{d}_{\mathrm{H}}$ even at the highest concentration (see Table 1).

Magnetization $M H$ curve for each particle batch was measured in an applied field varying from $-10 \mathrm{kOe}$ to $10 \mathrm{kOe}$ using alternating gradient magnetometer (AGM) at room temperature (Fig. 2). None of the sample exhibited hysteresis or coercivity, and therefore display superparamagnetic properties. Magnetization was normalized by the mass of iron resulting in comparable saturation magnetization values (approximately $75 \mathrm{emu} / \mathrm{g} \mathrm{Fe}$ ) for the small spheres, the cubes and the big spheres. The fact that the magnetization was found to be independent of the concentration for these three particle types supports the TDA results, where colloidal stability was detected. The ellipsoidal SPIONs, however, displayed different behavior. Overall, the saturation magnetization at low concentration is already significantly lower compared to the other three particle batches $(13 \mathrm{emu} / \mathrm{g}$ Fe to $\sim 75 \mathrm{emu} / \mathrm{g} \mathrm{Fe})$. This can be explained with the crystalline structure of the particles as well as the particle shape. It was reported earlier that ellipsoidal magnetite nanoparticles show lower magnetization profiles compared to spherical ones due to increased surface spin canting effects [40]. Additionally, XRD reveals that the ellipsoidal SPIONs still contain some wüstite, in contrast to the crystal lattices of the small, the large spheres, and the cubic particles, which are composed entirely of magnetite/maghemite (see Fig. 3). Due to the synthetic process (thermal decomposition of iron oleate complex), the bigger ellipsoidal particles contain paramagnetic impurities in the crystalline structure while the smaller ones are made entirely of magnetite/maghemite [45]. We tried to optimize our oxidation process to obtain these ellipsoidal particles without a wüstite phase. This was, probably due to the large particle sizes, not possible even after several consecutive oxidation procedures. We decided to continue this study using the present ellipsoidal SPIONs since detectable heating signals were obtained and no change in the collective particle behavior due to the presence of wüstite was expected. A drop in magnetization for ellipsoids at $80 \mathrm{mg} / \mathrm{mL}$ could be observed. Interestingly, the obvious explanation of particle-aggregation being responsible for the decrease in magnetization, which is commonly reported in the literature [46], is ruled out by TDA measurements which showed stable hydrodynamic diameters at both low and high concentrations (see Table 1). While our results suggest that factors like crystallographic impurity, polydispersity in size, aspect ratio and magnetic coupling

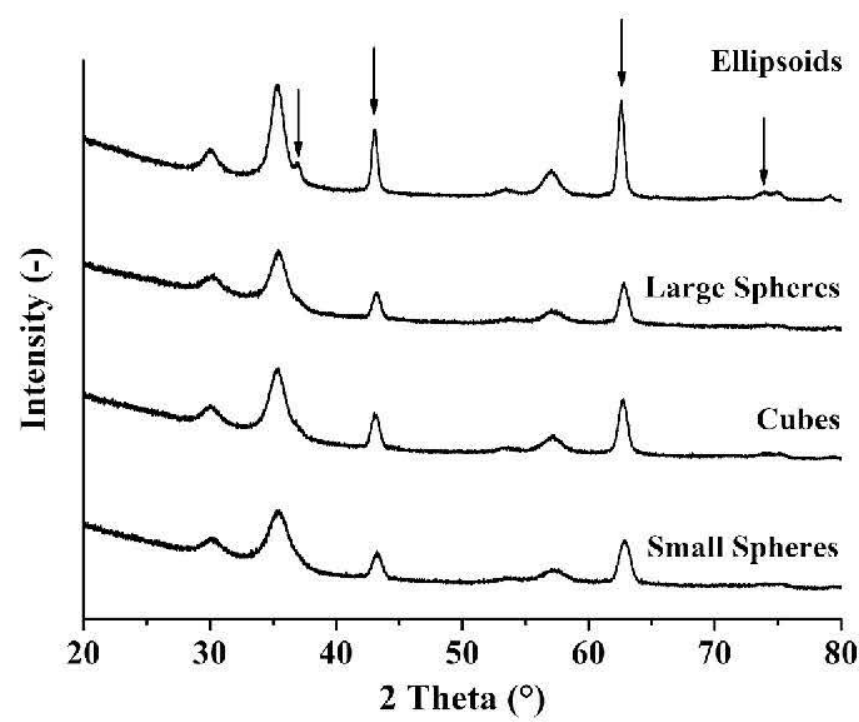

Fig. 3. Normalized XRD patterns of CA coated SPIONs. The curves are shifted vertically for the sake of visibility. Ellipsoids show a second small peak at $\sim 36^{\circ}$, higher peaks at $\sim 43^{\circ}$ and $\sim 63^{\circ}$, as well as a double peak at $\sim 74^{\circ}$ in comparison to small spheres, cubes and large spheres, indicating that wüstite is still present in the crystal lattice of the ellipsoids. 

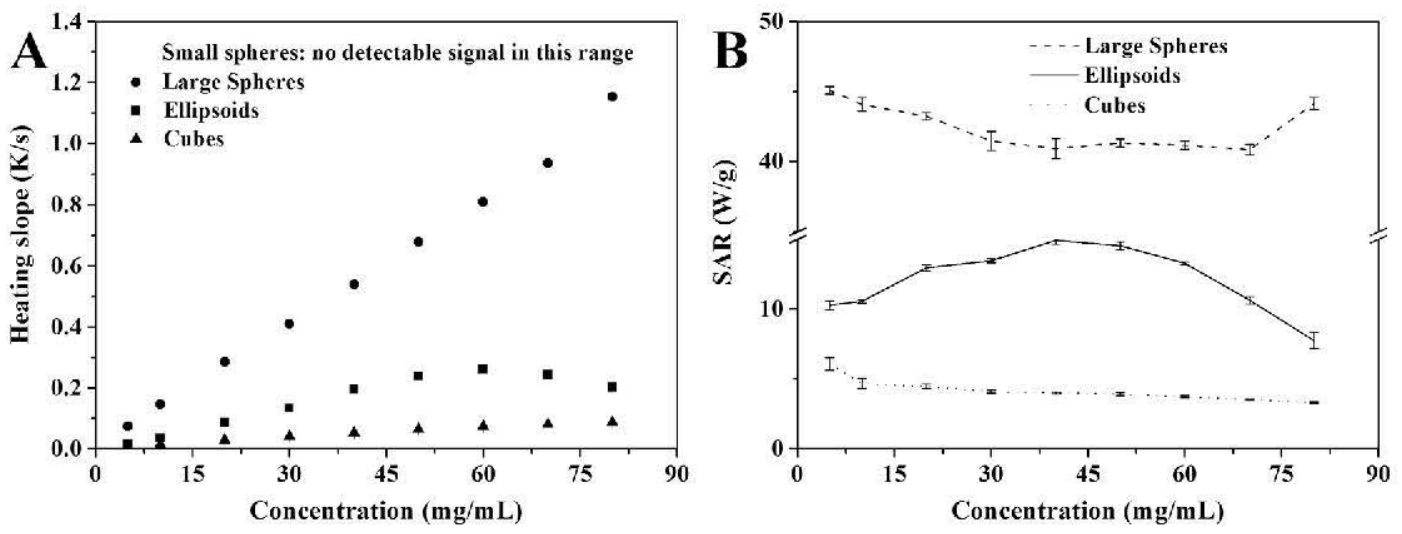

Fig. 4. A) Measurements of heating slope as a function of SPIONs concentration for the different particles and B) Calculated SAR values as a function of particle concentration. A) Data points represent triplicate experiments for cubes, large spheres and ellipsoids. Heating slope of small spheres was measured in a different concentration range due to low heating efficiency (see SI). All particles exhibit a linear trend in the heating slope, except ellipsoidal SPIONs showing a decrease at higher concentrations starting from $50 \mathrm{mg} / \mathrm{mL}$. B) (Note the break in y-axis) Error bars represent SAR calculation of triplicate heating slopes measurements. Lines are included as a guide for eyes. Linearity of heating slope result in stable SAR values for all 4 particles analyzed (SAR of small spheres is attached in SI). Only ellipsoids display a decreasing trend at higher concentration, due to deviation non-linear heating slope in this regime.

effects play an important role in the heating efficiency of these ellipsoidal particles at higher concentrations, further studies are needed to decipher the exact rationale for the loss in heating efficiency.

The thermal behavior was subsequently analyzed by LIT, which has the advantage of using small sample volumes $(15 \mu \mathrm{L})$ [31]. Laboratoryscale SPION synthesis is not suitable for providing large volumes of high concentrated particle dispersions required for fiber optic measurements [47]. Samples were up-concentrated to $80 \mathrm{mg} / \mathrm{mL}$ and diluted to eight different concentrations. It is well known that the heating power of SPIONs is strongly influenced by its core diameter [42], and therefore the small spheres did not show a detectable thermal signal in the range from 5 to $80 \mathrm{mg} / \mathrm{mL}$. To obtain measurable heating slopes for the $11 \mathrm{~nm}$ spheres, we further up-concentrated to $585 \mathrm{mg} / \mathrm{mL}$ and diluted to three concentrations (see supplementary information SI).

Fig. 4 A shows the strong linear character of the heating slopes for cubes and larges spheres independent of particle concentration. Normalizing the heat dissipated by the particles per mass of iron results in the specific absorption rate (Fig. 4 B). Both particle types display only small variations in their SAR values, which remains overall unchanged. The same behavior can be observed for small spheres at concentrations between 300 and $585 \mathrm{mg} / \mathrm{mL}$ where a heating signal was detectable (see SI). This leads to the assumption that the SAR for colloidally stable particles is independent of increasing concentrations for a wide range of sizes at a field frequency of $523 \mathrm{kHz}$. These results are perfectly in line with the findings of de la Presa et al. [48], which investigated concentrations between 6 and $300 \mathrm{mg} / \mathrm{mL}$ and find stable SAR values for three different particle sizes.

Contradictory to its size, the heating slope of the ellipsoidal particles was found to be lower compared to the large spheres, due to the presence of wüstite in the crystal lattice (see Fig. 3) as well as the spin canting effects [43]. The heating slopes of the ellipsoids show the same linear trend in the concentration range from $5 \mathrm{mg} / \mathrm{mL}$ to $50 \mathrm{mg} / \mathrm{mL}$ as large spheres and cubes. By further increasing the concentration, one can observe a decrease of the heating slope which is related to the lower magnetization (Fig. 2). Again, this decrease is independent from colloidal stability and must arise from an increasing collective behavior of the particles [2]. This data suggests that the SAR value of colloidal stable particles in water remains unchanged with increasing concentrations, independent of their size and shape. Other interactions such as, for example, collective magnetic behavior might have a strong influence on the magnetization and result in decreasing SAR with increasing concentrations. Further studies probing these interactions are consequently of particular importance.

\section{Conclusion}

We have presented the heating profiles of colloidally stable SPIONs up to clinically used concentrations via lock-in thermography. Nanoparticles were synthesized in house by thermal decomposition, covering a wide range of sizes ( $11 \mathrm{~nm}$ up to $22 \mathrm{~nm}$ ) and most common particle shapes (spherical, cubic and ellipsoidal), allowing the systematic study of the influence of size, shape and concentration on the particles heating efficiency. SPIONs were found to be colloidally stable at highest screened concentrations and display superparamagnetic behavior. Small spheres, cubes, and large spheres, consisting of magnetite/maghemite, exhibit a linear trend in their heating slope resulting in SAR values independent from size, shape and increasing concentrations. Ellipsoids showed lower magnetization due to presence of wüstite, however they display the same linear trend in heating slopes up to $50 \mathrm{mg} / \mathrm{mL}$, leading to unchanged SAR values. A further increase in the concentration generated lower magnetization and subsequently decreasing heating efficiency. Causes for this behavior remain unclear and need further investigation. In the context of using SPIONs in clinical hyperthermia treatment, colloidal stability is of utmost importance, as aggregates significantly lower AMF-induced SPION heating. Thus, more studies should focus on colloidal stability at high concentration, necessary to generate the desired temperatures in cancer tissue.

\section{Acknowledgements}

The authors gratefully acknowledge the financial support from the Swiss National Science Foundation (159803), the University of Fribourg and the Adolphe Merkle Foundation. Parts of this work were supported by the National Center of Competence in Research (NCCR) for BioInspired Materials. Access to AGM was kindly provided by the Institute for Geophysics of the ETH Zurich. Utilization of XRD was amicably earmarked by the Unit of Earth Sciences, Department of Geosciences of the University of Fribourg.

\section{Appendix A. Supplementary data}

Supplementary data to this article can be found online at https:// doi.org/10.1016/j.jmmm.2018.10.009.

\section{References}

[1] S. Laurent, S. Dutz, U.O. Häfeli, M. Mahmoudi, Magnetic fluid hyperthermia: Focus on superparamagnetic iron oxide nanoparticles, Adv. Colloid Interface Sci. 166 (1-2) 
(2011) 8-23.

[2] A.E. Deatsch, B.A. Evans, Heating efficiency in magnetic nanoparticle hyperthermia, J. Magn. Magn. Mater. 354 (2014) 163-172.

[3] A.K. Gupta, M. Gupta, Cytotoxicity suppression and cellular uptake enhancement of surface modified magnetic nanoparticles, Biomaterials 26 (13) (2005) 1565-1573.

[4] R.E.E. Rosensweig, Heating magnetic fluid with alternating magnetic field, J. Magn. Magn. Mater. 252 (2002) 370-374.

[5] R. Hergt, S. Dutz, R. Müller, M. Zeisberger, Magnetic particle hyperthermia: nanoparticle magnetism and materials development for cancer therapy, J. Phys. Condens. Matter 18 (38) (2006) S2919-\$2934.

[6] C.R. Kumar, F. Mohammad, Magnetic nanomaterials for hyperthermia-based therapy and controlled drug delivery, Adv. Drug Deliv. Rev. 63 (9) (2011) 789-808.

[7] B. Mehdaoui, A. Meffre, J. Carrey, S. Lachaize, L.M. Lacroix, M. Gougeon, B. Chaudret, M. Respaud, Optimal size of nanoparticles for magnetic hyperthermia: A combined theoretical and experimental study, Adv. Funct. Mater. 21 (23) (2011) $4573-4581$.

[8] C.R. Gilchrist, R. Medal, W.D. Shorey, R.C. Hanselman, J.C. Parrott, Selective Inductive Heating of Lymph Nodes, Ann. Surg. 146 (4) (1957) 596-606.

[9] P. Lemal, C. Geers, B. Rothen-Rutishauser, M. Lattuada, A. Petri-Fink, Measuring the heating power of magnetic nanoparticles: an overview of currently used methods, Mater. Today Proc. 4 (2017) S107-\$117.

[10] K. Maier-Hauff, F. Ulrich, D. Nestler, H. Niehoff, P. Wust, B. Thiesen, H. Orawa V. Budach, A. Jordan, Efficacy and safety of intratumoral thermotherapy using magnetic iron-oxide nanoparticles combined with external beam radiotherapy on patients with recurrent glioblastoma multiforme, J. Neurooncol. 103 (2) (2011) 317-324.

[11] J.G. Ovejero, D. Cabrera, J. Carrey, T. Valdivielso, G. Salas, F.J. Teran, Effects of inter- and intra-aggregate magnetic dipolar interactions on the magnetic heating efficiency of iron oxide nanoparticles, Phys. Chem. Chem. Phys. 18 (16) (2016) 10954-10963.

[12] A. Espinosa, R. Di Corato, J. Kolosnjaj-Tabi, P. Flaud, T. Pellegrino, C. Wilhelm, Duality of iron oxide nanoparticles in cancer therapy: amplification of heating efficiency by magnetic hyperthermia and photothermal bimodal treatment, ACS Nano 10 (2) (2016) 2436-2446.

[13] G. Kandasamy, A. Sudame, P. Bhati, A. Chakrabarty, D. Maity, Systematic investigations on heating effects of carboxyl-amine functionalized superparamagnetic iron oxide nanoparticles (SPIONs) based ferro fluids for in vitro cancer hyperthermia therapy, J. Mol. Liq. 256 (2018) 224-237.

[14] Y. Luengo, V. Velasco, M.P. Morales, M. Iglesias, P. Crespo, A. Hernando, Particle interactions in liquid magnetic colloids by zero field cooled measurements: effects on heating efficiency, J. Phys. Chem. C 119 (2015) 11022-11030.

[15] C. Blanco-Andujar, D. Ortega, P. Southern, S.A. Nesbitt, N.T.K. Thanh, Q.A. Pankhurst, Real-time tracking of delayed-onset cellular apoptosis induced by intracellular magnetic hyperthermia, Nanomedicine (Lond.) 11 (2) (2016) $121-136$

[16] J. Wan, R. Yuan, C. Zhang, N. Wu, F. Yan, S. Yu, Stable and biocompatible colloidal dispersions of superparamagnetic iron oxide nanoparticles with minimum aggregation for biomedical applications, J. Phys. Chem. C 120 (2016) 23799-23806.

[17] N.G. Shetake, A. Kumar, S. Gaikwad, P. Ray, S. Desai, R.S. Ningthoujarn, R.K. Vatsa, B.N. Pandey, Magnetic nanoparticle-mediated hyperthermia therapy induces tumour growth inhibition by apoptosis and Hsp90/AKT modulation, Int. J. Hyperth. 31 (8) (2015) 909-919.

[18] D. Sakellari, K. Brintakis, A. Kostopoulou, E. Myrovali, K. Simeonidis, A. Lappas, M. Angelakeris, Ferrimagnetic nanocrystal assemblies as versatile magnetic particle hyperthermia mediators, Mater. Sci. Eng. C 58 (2016) 187-193.

[19] S.G. Mendo, A.F. Alves, L.P. Ferreira, M.M. Cruz, M.H. Mendonça, M. Godinho, M.D. Carvalho, Hyperthermia studies of ferrite nanoparticles synthesized in the presence of cotton, New J. Chem. 39 (2015) 7182-7193.

[20] A.S. Eggeman, S.A. Majetich, D. Farrell, Q.A. Pankhurst, Size and concentration effects on high frequency hysteresis of iron oxide nanoparticles, IEEE Trans. Magn. 43 (6) (2007) 2451-2453.

[21] J.L. Dormann, D. Fiorani, J. Tholence, Journal of Physics C: Solid State Physics Related content A dynamic study of small interacting particles: superparamagnetic model and spin-glass laws A dynamic study of small interacting particles: superparamagnetic model and spin-glass laws, J. Phys. Chem. C 21 (1988) 2015-2034.

[22] S. Morup, E. Tronc, Superparamagnetic relaxation of weakly interacting particles, Phys. Rev. Lett. 72 (20) (1994) 3278-3281.

[23] A. Urtizberea, E. Natividad, A. Arizaga, M. Castro, A. Mediano, Specific absorption rates and magnetic properties of ferrofluids with interaction effects at low concentrations, J. Phys. Chem. C 114 (2010) 4916-4922.

[24] Y. Piñeiro-Redondo, M. Bañobre-López, I. Pardiñas-Blanco, G. Goya, M.A. LópezQuintela, J. Rivas, The influence of colloidal parameters on the specific power absorption of PAA-coated magnetite nanoparticles, Nanoscale Res. Lett. 6 (383) (2011) 1-7.

[25] X. Wang, H. Gu, Z. Yang, The heating effect of magnetic fluids in an altemating magnetic field, J. Magn. Magn Mater. 293 (2005) 334-340.

[26] T. Thu, T. Mai, P.T. Ha, P.H. Linh, N.C. Thuan, Magnetic fluid based on Fe304 nanoparticles: preparation and hyperthermia application, J. Phys. Conf. Ser. 187 (2009) 6-11.

[27] Navid Z. Gao, J. Zhang, H.L. Ring, Q. Shao, F. Liu, M. Mcdermott, A. Fok, Y. Rabin, K.G.M. Brockbank, M. Garwood, C.L. Haynes, J.C. Bischof, Improved tissue cryopreservation using inductive heating of magnetic nanoparticles, Sci. Trasnlational. Med. 9 (2017).

[28] C.L. Dennis, A.J. Jackson, J.A. Borchers, R. Ivkov, A.R. Foreman, J.W. Lau, E. Goemitz, C. Gruettner, The influence of collective behavior on the magnetic and heating properties of iron oxide nanoparticles, J. Appl. Phys. 103 (2008) p. 07A3019-1-4.

[29] M. Andrés Vergés, R. Costo, G.A. Roca, J.F. Marco, G.F. Goya, C.J. Serna, M.P. Morales, Uniform and water stable magnetite nanoparticles with diameters around the monodomain-multidomain limit, J. Phys. D. Appl. Phys. 41 (2008) 10.

[30] S. Ota, T. Yamada, Y. Takemura, Dipole-dipole interaction and its concentration dependence of magnetic fluid evaluated by alternating current hysteresis mea surement, J. Appl. Phys. 713 (2016).

[31] C.A. Monnier, M. Lattuada, D. Burnand, F. Crippa, J.C. Martinez-Garcia, A.M. Hirt B. Rothen-Rutishauser, M. Bonmarin, A. Petri-Fink, A lock-in-based method to examine the thermal signatures of magnetic nanoparticles in the liquid, solid and aggregated states, Nanoscale 8 (2016).

[32] J.-G.J.J.-H. Park, K. An, Y. Hwang, H.-J. Noh, J.-Y. Kim, N.-M. Hwang, T. Hyeon, Ultra-large-scale syntheses of monodisperse nanocrystals, Nat. Mater. $3(12)(2004)$ 891-895.

[33] Y. Hou, Z. Xu, S. Sun, Controlled synthesis and chemical conversions of FeO nanoparticles, Angew. Chemie - Int. Ed. 46 (33) (2007) 6329-6332.

[34] E. Wetterskog, M. Agthe, A. Mayence, J. Grins, Precise control over shape and size of iron oxide nanocrystals suitable for assembly into ordered particle arrays, Sci. Technol. Adv. Mater. 15 (2014) 055010.

[35] M. Lattuada, T.A. Hatton, Functionalization of monodisperse magnetic nanoparticles, Langmuir 23 (4) (2007) 2158-2168.

[36] M.E. de Sousa, M.B. Fernández van Raap, P.C. Rivas, P. Mendoza Zélis, P. Girardin, G.A. Pasquevich, J.L. Alessandrini, D. Muraca, F.H. Sánchez, Stability and relaxation mechanisms of citric acid coated magnetite nanoparticles for magnetic hyperthermia, J. Phys. Chem. C 117 (10) (2013) 5436-5445.

[37] B. Michen, C. Geers, D. Vanhecke, C. Endes, B. Rothen-Rutishauser, S. Balog, A. Petri-Fink, Avoiding drying-artifacts in transmission electron microscopy: characterizing the size and colloidal state of nanoparticles, Sci. Rep. 5 (2015) 9793.

[38] D. Bossert, F. Crippa, A. Petri-Fink, S. Balog, Hypothesis test of the photon count distribution for dust discrimination in dynamic light scattering, Anal. Chem. 90 (2018) 3656-3660.

[39] C.A. Monnier, F. Crippa, C. Geers, E. Knapp, B. Rothen-Rutishauser, M. Bonmarin, M. Lattuada, A. Petri-Fink, Lock-in thermography as an analytical tool for magnetic nanoparticles: measuring heating power and magnetic fields, J. Phys. Chem. C 121 (2017) 27164-27175.

[40] S. Balog, D.A. Urban, A.M. Milosevic, F. Crippa, B. Rothen-Putishauser, A. PetriFink, Taylor dispersion of nanoparticles, J. Nanopart. Res. 19 (287) (2017) 1-11.

[41] S. Balog, L. Rodriguez-Lorenzo, C.A. Monnier, M. Obiols-Rabasa, B. RothenRutishauser, P. Schurtenberger, A. Petri-Fink, Characterizing nanoparticles in complex biological media and physiological fluids with depolarized dynamic light scattering, Nanoscale 7 (14) (2015) 5991-5997.

[42] P. Lemal, C. Geers, C.A. Monnier, F. Crippa, L. Daum, D.A. Urban, B. RothenRutishauser, M. Bonmarin, A. Petri-Fink, T.L. Moore, Lock-in thermography as a rapid and reproducible themal characterization method for magnetic nanoparticles, J. Magn. Magn. Mater. (2016).

[43] J. Choi, J. Cha, J.-K. Lee, Synthesis of various magnetite nanoparticles through simple phase transformation and their shape-dependent magnetic properties, RSC Adv. 3 (2013) 8365-8371.

[44] F. Crippa, T.L. Moore, M. Mortato, C. Geers, L. Haeni, Ann M. Hirt, Barbara RothenRutishauser, Alke Petri-Fink, Dynamic and biocompatible thermo-responsive mag netic hydrogels that respond to an alternating magnetic field, J. Magn. Magn. Mater. 427 (2016) 212-219 2017.

[45] A. Lak, M. Kraken, F. Ludwig, A. Kornowski, D. Eberbeck, S. Sievers, F.J. Litterst, Size dependent structural and magnetic properties of $\mathrm{FeO}-\mathrm{Fe} 304$ nanoparticles, Nanoscale 5 (2013) 12286-12295.

[46] C. Guibert, V. Dupuis, V. Peyre, J. Fresnais, Hyperthermia of magnetic nanoparticles: experimental study of the role of aggregation, J. Phys. Chem. C 119 (50) (2015) 28148-28154.

[47] S. Huang, S.-Y. Wang, A. Gupta, D.-A. Borca-Tasciuc, S.J. Salon, On the measurement technique for specific absorption rate of nanoparticles in an alternating electromagnetic field, Meas. Sci. Technol. 23 (2012) p. 035701-6pp.

[48] P. de la Presa, Y. Luengo, M. Multigner, R. Costo, M.P. Morales, G. Rivero, A. Hernando, Study of heating efficiency as a function of concentration, size, and applied field in $\gamma$-Fe203 nanoparticles, J. Phys. Chern. C 116 (2012) 25602-25610. 